\title{
Ceria Nanozymes with Preferential Renal Uptake for Acute Kidney Injury Alleviation
}

Dong-Yang Zhang ${ }^{1,2 \ddagger}$, Hengke Liu ${ }^{2 \ddagger}$, Chunying $\mathrm{Li}^{1 \ddagger}$, Muhammad Rizwan Younis ${ }^{2,3}$, Shan

Lei $^{2,3}$, Chen Yang ${ }^{2,3}$, Jing $\operatorname{Lin}^{2}$, Zhiming $\mathrm{Li}^{1 *}$, and Peng Huang ${ }^{1,2 *}$

1. Department of Dermatology and Venerology, the First Affiliated Hospital of Wenzhou Medical University, Wenzhou, Zhejiang 325000, China.

2. Marshall Laboratory of Biomedical Engineering, International Cancer Center, Laboratory of Evolutionary Theranostics (LET), School of Biomedical Engineering, Shenzhen University Health Science Center, Shenzhen, 518060, China.

3. Key Laboratory of Optoelectronic Devices and Systems of Ministry of Education and Guangdong Province, College of Optoelectronic Engineering, Shenzhen University, Shenzhen 518060, China.

*Corresponding author: zhi-mingli@163.com (Z. L.); peng.huang@ szu.edu.cn (P.H.).

+ Equal contribution authors. 


\section{Materials}

Cerium nitrate pentahydrate and MTT were obtained from J\&K Reagent (China). Citric acid, ammonia solution, TMB and Cyt $\mathrm{C}$ were bought from Aladdin Reagent (China). Hydrogen peroxide $\left(\mathrm{H}_{2} \mathrm{O}_{2}, 30 \%\right)$, nitric acid, and hydrochloric acid (36\%) were obtained from Guangzhou Chemical Reagent Factory. ABTS and DCF were bought from Sigma-Aldrich (USA). Mito-Tracker Green assay kit and Apoptosis Detection kit (propidium iodide/annexin V-FITC) were obtained from Beyotime Institute of Biotechnology (China). The Fluorimetric Peroxynitrite Quantification Kit was obtained from AAT Bioquest (USA).

\section{Instrumentation}

TEM images were obtained using a transmission electron microscopy (JEM-2100F, JEOL, Japan) at $200 \mathrm{keV}$. The hydrophilic diameter of the nanoparticles was determined by a dynamic laser scattering (DLS) particle size analyzer (Malvern 2000, USA). An X-ray diffractometer (PANalytical Empyrean, Netherlands) was used to analyze crystal structure of the nanoparticles. FT-IR spectra were taken between $500-5000 \mathrm{~cm}^{-1}$ by using Perkin Elmer (L16000300 Spectrum TWO LITA, Llantrisant, UK). An X-ray photoelectron spectrometer (K-Alpha+, Thermo Fisher Scientific, UK) was used to measure XPS spectra of ceria NPs before and after oxidized by $\mathrm{H}_{2} \mathrm{O}_{2}$. The content of Ce was detected by ICP-MS (PerkinElmer, NexIon300X, USA). The absorption spectra were detected by an absorption spectrophotometer (Cary 60, Agilent Technologies, USA). The cellular fluorescence imaging was conducted using a confocal fluorescence microscopy (LSM880, ZEISS, Germany).

\section{$\mathrm{H}_{2} \mathrm{O}_{2}$ scavenging activity of ceria NPs}

The Hydrogen Peroxide Detection Kit (Nanjing Jiancheng Bioengineering Institute, China) was utilized to detect the $\mathrm{H}_{2} \mathrm{O}_{2}$ scavenging ability of ceria NPs with various concentrations. The absorbance peak $(405 \mathrm{~nm})$ of yellow complex was measured, formed by reaction of $\mathrm{H}_{2} \mathrm{O}_{2}$ and ammonium molybdate. Different concentrations of ceria NPs $(100-400 \mu \mathrm{g} / \mathrm{mL})$ were mixed with $\mathrm{H}_{2} \mathrm{O}_{2}(2 \mathrm{mM})$ at $37{ }^{\circ} \mathrm{C}$ for $1 \mathrm{~h}$. Afterwards, the content of remaining $\mathrm{H}_{2} \mathrm{O}_{2}$ along with $\mathrm{H}_{2} \mathrm{O}_{2}$ inhibition rate was 
estimated.

\section{-OH scavenging activity of ceria NPs}

The hydroxyl radical detection kit (Cell Biolabs, Inc., USA, HORAC) was used to analyze the hydroxyl radical scavenging activity of ceria NPs. fluorescent solution $(140 \mu \mathrm{L})$ was incubated with samples $(20 \mu \mathrm{L})$ at different concentrations $(0-400$ $\mu \mathrm{g} / \mathrm{mL})$. Next, $20 \mu \mathrm{L}$ of a hydroxyl radical initiator and Fenton reagent was added to each well according to priority. The plate was incubated at $25{ }^{\circ} \mathrm{C}$ for $30 \mathrm{~min}$. Finally, the fluorescence intensity was measured by a microplate reader.

\section{ABTS radical cations scavenging activity of ceria NPs}

A discoloration test of the ABTS radical cations was utilized to measure the free radical scavenging capacity of ceria NPs. First, ABTS radical cations $\left(\bullet \mathrm{ABTS}^{+}\right)$was produced by mixing ABTS aqueous solution $(7 \mathrm{mM})$ and potassium persulfate $(2.45$ $\mathrm{mM}$ ) for $14 \mathrm{~h}$. Then, different concentrations of ceria NPs (50-400 $\mu \mathrm{g} / \mathrm{mL})$ were mixed to the above mixture. Finally, the absorbance value at $734 \mathrm{~nm}$ of the final solutions was measured and removal efficiency of ABTS is calculated as follows: $\left[\left(A_{B}-A_{C}\right) / A_{B}\right]^{*} 100 . A_{B}$ : the absorbance of $\bullet A_{B T S}^{+}$pure solution; $A_{C}$ : the absorbance of $\bullet \mathrm{ABTS}^{+}$solution mixed with ceria NPs.

\section{$\cdot \mathrm{O}_{2}{ }^{-}$scavenging with ceria NPs}

SOD Analysis Kit (Sigma-Aldrich, USA) was utilized to evaluate the $\cdot \mathrm{O}_{2}{ }^{-}$scavenging ability of ceria NPs. $20 \mu \mathrm{L}$ sample with various concentrations of ceria NPs $(0-100$ $\mu \mathrm{g} / \mathrm{mL}$ ) was added to each well. After adding $160 \mu \mathrm{L}$ 2-(4-iodophenyl)-3-(4-nitrophenyl)-5-(2,4-disulfophenyl)-2H-tetrazole sodium (WST-1) to each well, xanthine oxidase solution $(20 \mu \mathrm{L})$ was sequentially mixed in the microtiter plate. The plate was then incubated at $37^{\circ} \mathrm{C}$ for $20 \mathrm{~min}$. Finally, the absorbance intensity $(450 \mathrm{~nm})$ was detected using a microplate reader.

\section{Cyt C test}

Ceria NPs $(100 \mu \mathrm{g} / \mathrm{mL})$ were added to Cyt $\mathrm{C}(500 \mu \mathrm{M})$ in PBS at $\mathrm{pH}$ 7.4. Then, the above solution was incubated for $1 \mathrm{~h}$ in the dark at room temperature and detected by absorption spectrometer. To get rid of oxygen, the solutions were pumped with nitrogen for $1 \mathrm{~h}$ and the above experiment was repeated. 


\section{Cell culture}

HEK293T cells were obtained from the OBiO Technology Corp., Ltd (Shanghai, China). The Dulbecco's modified Eagle's Medium (DMEM) media were bought from Wisent Inc. The cells were cultured in DMEM (Gibco, BRL) and supplemented with $1 \%$ penicillin/streptomycin and $10 \%$ fetal bovine serum at $37{ }^{\circ} \mathrm{C}$ under $5 \% \mathrm{CO}_{2}$.

\section{Glycerol-induced AKI mouse model}

All female BALB/c mice (4-6 weeks, 17 21 g) were purchased from Guangdong Medicinal Laboratory (China) and all animal studies were carried out according to the protocol approved by the Animal Ethics Committee of the Center of Experiment Animals of the University of Shenzhen.

AKI model induced by glycerol: All BALB/c mice were deprived of water but had access to food for $15 \mathrm{~h}$, when the mice hind limbs received an intramuscular (i.m.) injection of $50 \%(\mathrm{v} / \mathrm{v})$ glycerol $(8 \mathrm{ml} / \mathrm{kg})$. Subsequently, all mice had access to water and food. The AKI mouse model was successfully established after injection for $2 \mathrm{~h}$ and used for further experiments.

\section{Statistical analysis}

The quantitative data analysis was manifested as mean \pm standard deviation. All biological tests were conducted at least twice. Values with $\mathrm{P}<0.05$ were considered statistically significant $(* \mathrm{P}<0.05, * * \mathrm{P}<0.01, * * * \mathrm{P}<0.001$, ns: non-significant). 


\section{Supporting Figures}

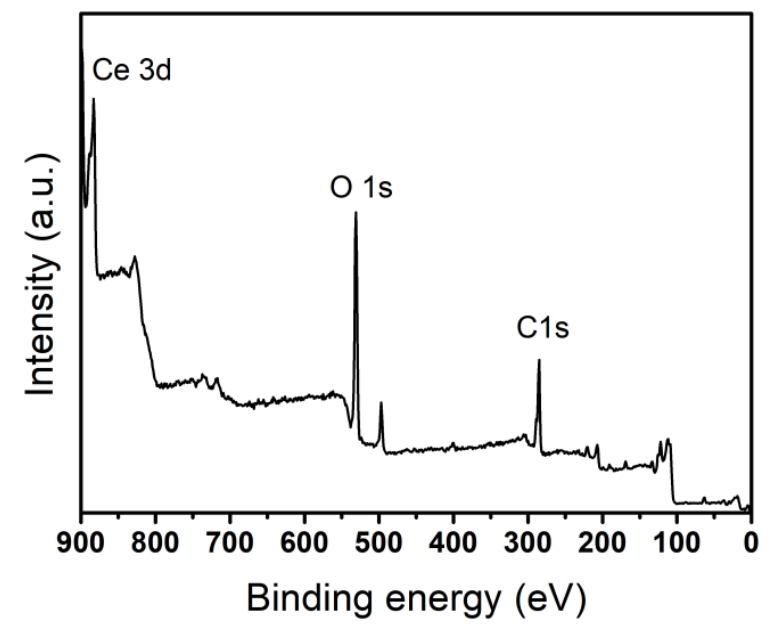

Figure. S1 XPS spectrum of ceria NPs.
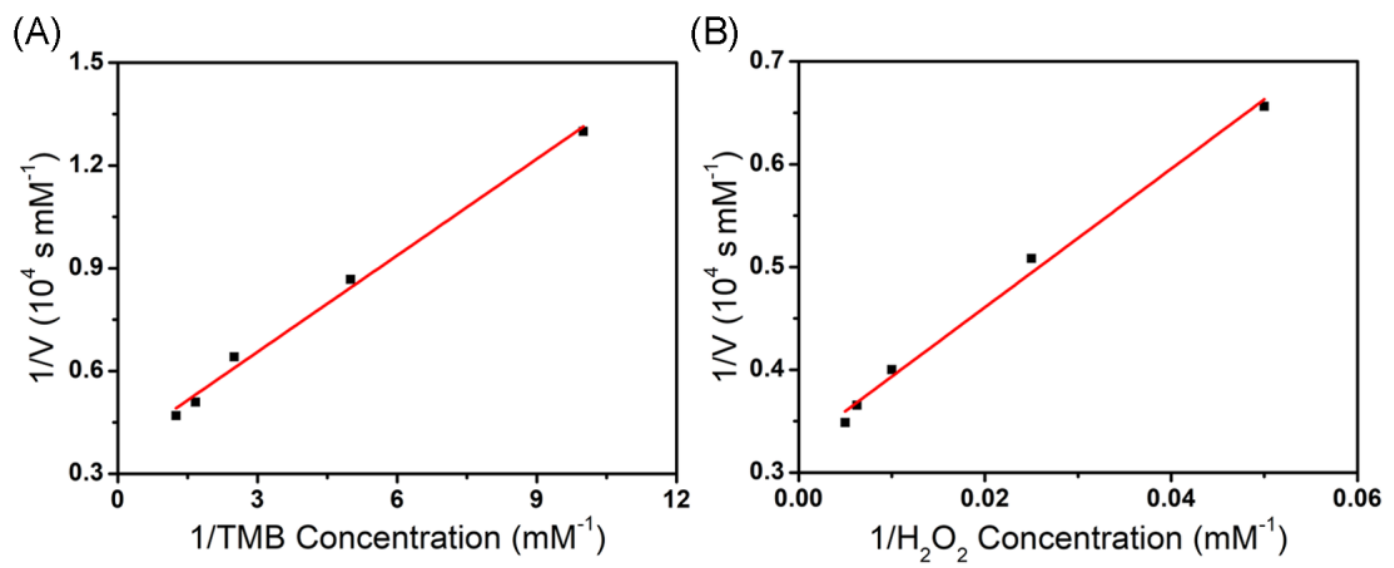

Figure. S2 (A, B) Double-reciprocal plots of the activity of ceria NPs, corresponding to Figure. $2 \mathrm{~B}$ and $2 \mathrm{C}$. 


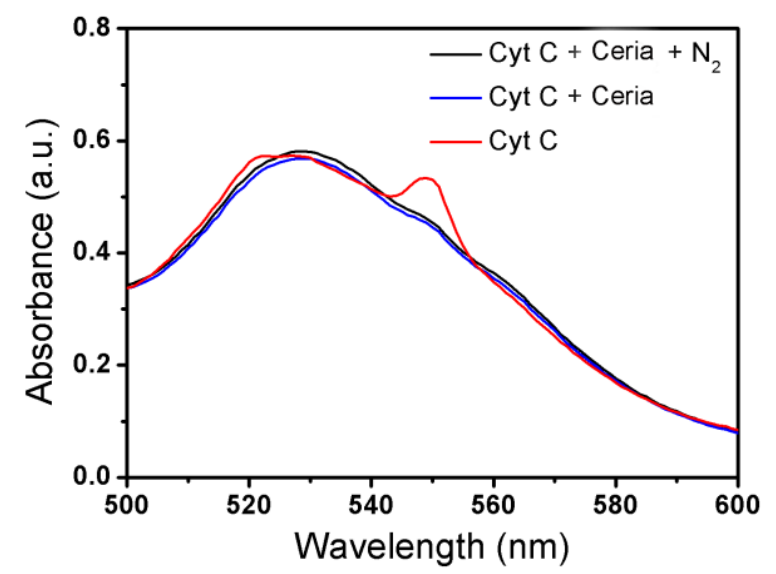

Figure. S3 Absorbance spectra of original Cyt C (red line), Cyt C reacted with ceria NPs (blue line) (black line), and Cyt C reacted with ceria NPs under deoxygenated condition from 450 to $600 \mathrm{~nm}$.

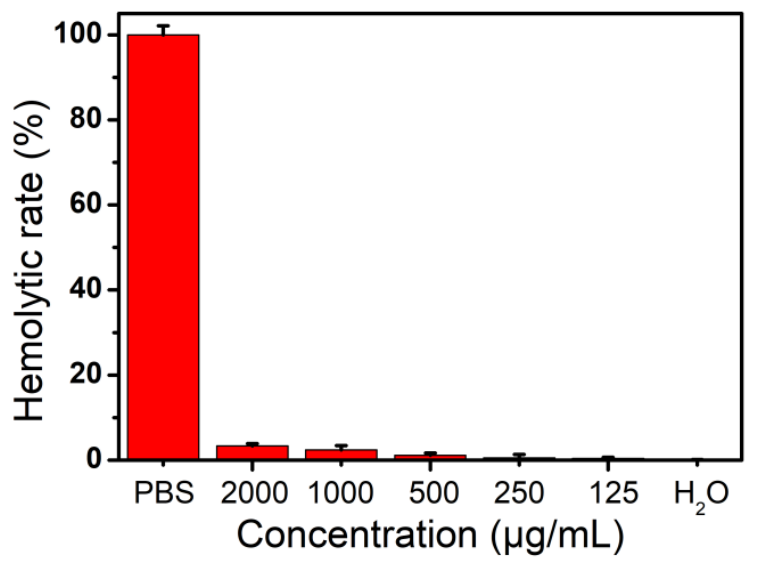

Figure. S4 Hemolysis test of ceria NPs on red blood cells.

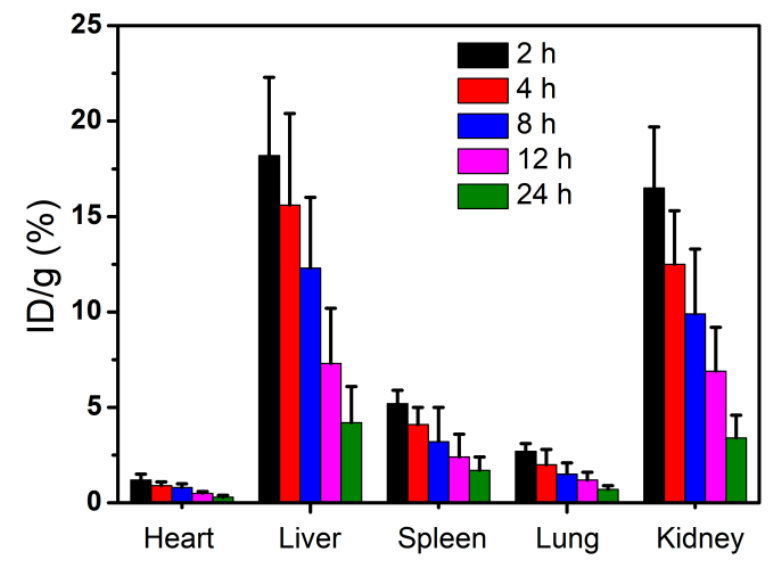

Figure. S5 ICP-MS analysis of the in vivo biodistribution of ceria NPs in major organs of healthy mice. 


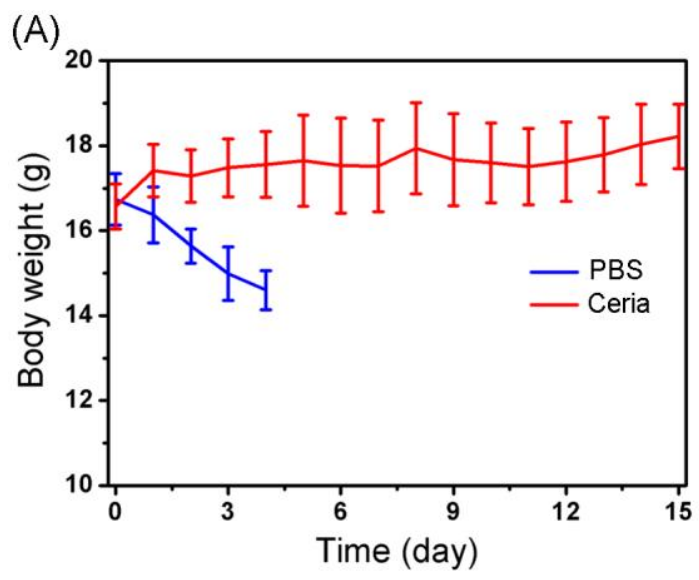

(B)
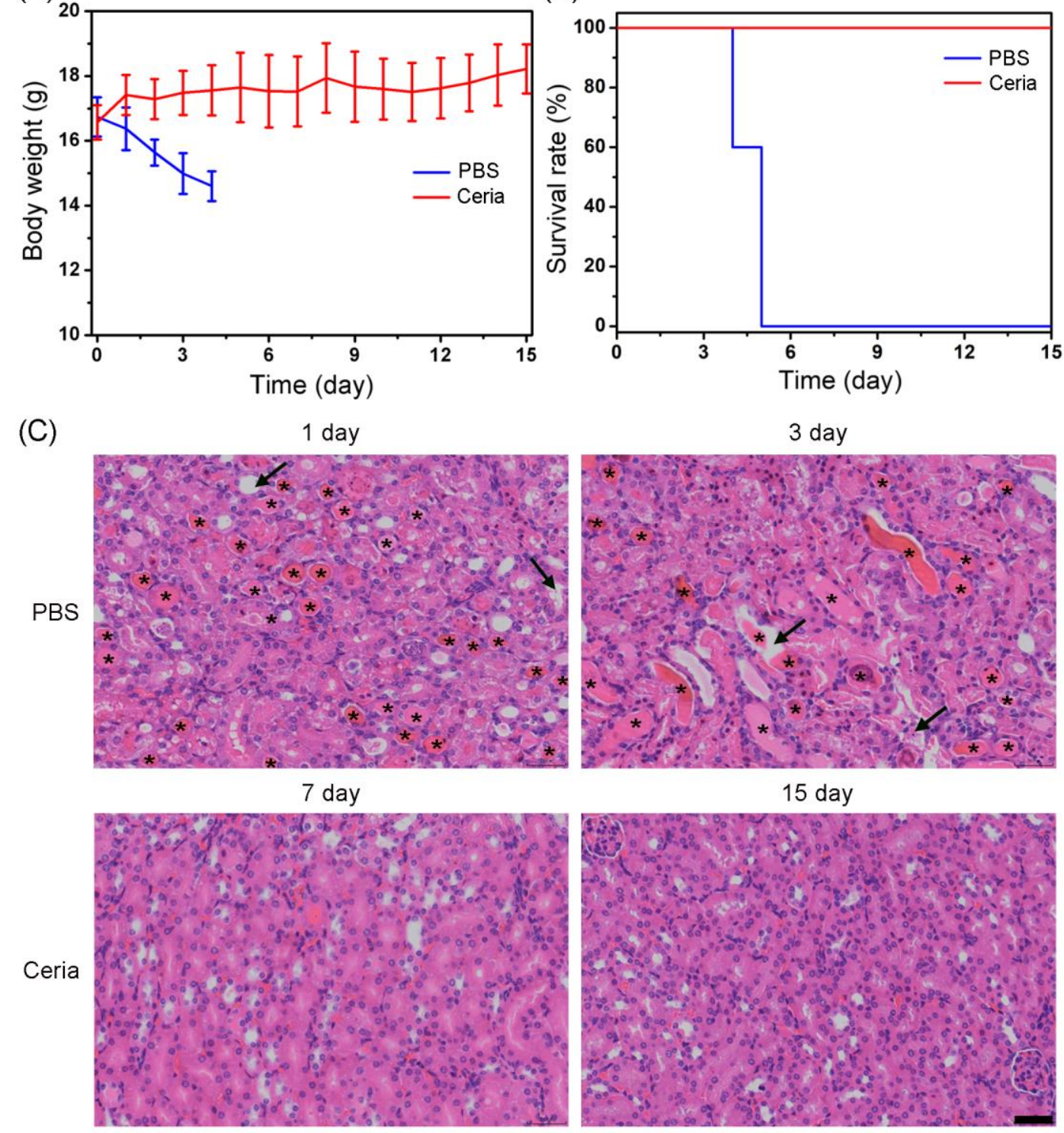

Figure. S6 (A) The body weight and (B) survival rate AKI mice in the 14 days following PBS or ceria NPs treatment ( $n=5$, mean \pm s.d.). (C) H\&E stained images of kidney tissues collected from PBS- or ceria NPs-treated mice. The scale bar is 50 $\mu \mathrm{m}$. Arrows indicate damaged tubules and asterisks indicate the formation of casts. 


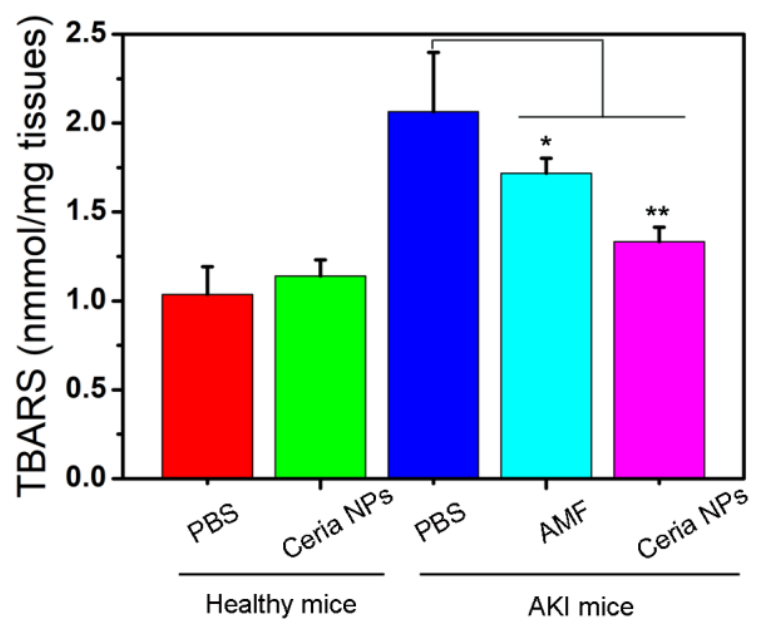

Figure. S7 The degree of lipid peroxidation detection. Measurement of thiobarbituric acid-reactive substances (TBARS), an index of lipid peroxidation, in the renal tissues homogenate collected from each group $(n=3$, mean \pm s.d. $)$.

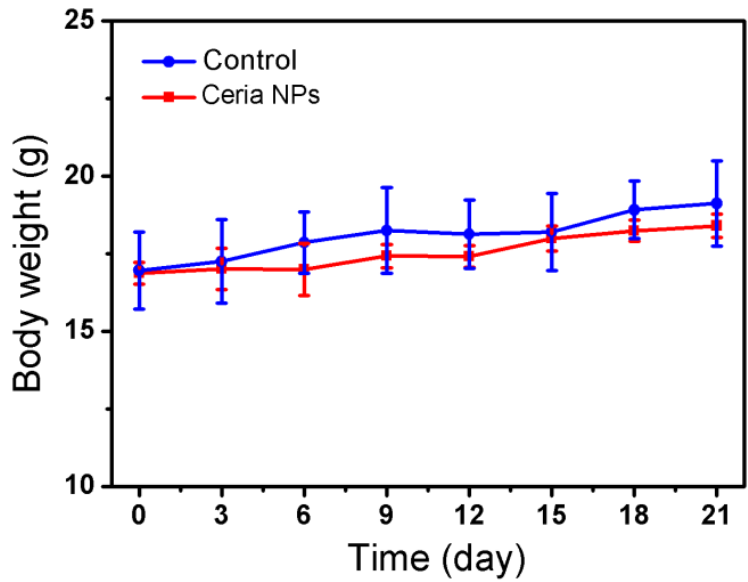

Figure. S8 Body weight changes after intravenous injection of ceria NPs or PBS. 